\title{
Design, synthesis and biological evaluation of isochroman-4-one hybrids bearing piperazine moiety as antihypertensive candidates
}

\author{
Shaowen Xie ${ }^{\mathrm{a}}$, Xinnan $\mathrm{Li}^{\mathrm{a}}$, Pengfei Zhanga ${ }^{\mathrm{a}}$, Jia Wang ${ }^{\mathrm{a}}$, Chaolei Wang ${ }^{\mathrm{a}}$, Shengtao $\mathrm{Xu}^{\mathrm{a}}$, \\ Zheng $\mathrm{Wu}^{\mathrm{a}}$, Jie Liu ${ }^{\mathrm{b}, *}$, Zheying $\mathrm{Zhu}^{\mathrm{c}}$, Jinyi $\mathrm{Xu}^{\mathrm{a}, *}$ \\ ${ }^{a}$ State Key Laboratory of Natural Medicines and Department of Medicinal Chemistry, China Pharmaceutical University, 24 \\ Tong Jia Xiang, Nanjing 210009, PR China \\ ${ }^{b}$ Department of Organic Chemistry, China Pharmaceutical University, 24 Tong Jia Xiang,Nanjing 210009, P. R. China \\ 'Division of Molecular Therapeutics \& Formulation, School of Pharmacy, The University of Nottingham, University Park Campus, \\ Nottingham NG7 2RD, UK
}

\begin{abstract}
Dihydroxy-3-methyl-isochromanone-4 (XJP), is a polyphenolic natural product with moderate antihypertensive activity. To obtain new agents with stronger potency and safer profile, we employed XJP and naftopidil as the lead compounds to design and synthesize a novel class of hybrids as antihypertensive candidates, In the present study, a series of hybrids (6a-r) of XJP bearing arylpiperazine moiety, which is identified as the pharmacophore of naftopidil, were designed and synthesized as novel $\alpha_{1}$-adrenergic receptor antagonists. The biological evaluation showed that target compounds $6 \mathbf{c}, 6 \mathbf{6}, \mathbf{6 f}, \mathbf{6 g}, \mathbf{6 h}, \mathbf{6 m}$ and $\mathbf{6 q}$ possessed potent in vitro vasodilation potency and $\alpha_{1}$-adrenergic receptor antagonistic activity. Furthermore, the most potent compound 6e significantly reduced the systolic and diastolic blood pressure in spontaneously hypertensive rats (SHRs), which was comparable to that of naftopidil, and it had no observable effects on the basal heart rate, suggesting that $6 \mathbf{e}$ deserves to be further investigated as a potential clinical candidate for the treatment of hypertension.
\end{abstract}

${ }^{*}$ Corresponding author. Tel.: +86-25-83271299; fax: +86-25-83302827; e-mail:cpu-jill@163.com; jinyixu@china.com 
Keywords: antihypertensive activity, $\alpha_{1}$-adrenergic receptor antagonist, isochroman-4one, hybrids, arylpiperazine

\section{Introduction}

Hypertension has become one of the most prevalent health issues in 21st century. Long term high blood pressure is a major risk factor for coronary artery disease, stroke, heart failure, and other cardiovascular diseases ${ }^{1,2}$. The conventional treatment of hypertension involves combination of drugs including angiotensin II receptor antagonists, calcium channel blockers, angiotensin-converting enzyme inhibitors (ACEI), $\alpha_{1}$-adrenergic receptor antagonists, $\beta$-adrenergic receptor blockers, diuretics, etc $^{3,4,5,6}$. Despite various drugs have been applied in clinic for the treatment of hypertension, effective blood pressure control remains a major medical challenge and there has been a consistent demand for more effective and safer antihypertensive $\operatorname{drugs}^{7,8}$. Hence, development of new drugs with multiple actions could be of great significance for the treatment of hypertension.

( \pm )-7,8-Dihydroxy-3-methyl-isochromanone-4[( \pm -XJP, Figure 1, 1], isolated from the banana (Musasapientum L.) peel extract, is a structurally unique polyphenolic compound with multiple biological activities including antioxidative, antihypertensive and cardioprotective activities ${ }^{9,10}$. Particularly, in our previous studies, $( \pm)$-XJP exhibited moderate antihypertensive activity in a dose-dependent manner, the maximum antihypertensive effect of $( \pm)$-XJP at the dose of $100 \mathrm{mg} / \mathrm{kg}$ was comparable to that of captopril at the dose of $25 \mathrm{mg} / \mathrm{kg}$ in renal hypertensive rats (RHRs) ${ }^{11}$. In the further structural modification of ( \pm )-XJP, some analogues (Figure 1, 2-4) were found to be more potent than $( \pm)$-XJP in spontaneously hypertensive rats (SHRs) ${ }^{12}$.

$\alpha_{1}$-Adrenergic receptor antagonists have been used for the treatment of hypertension since 1976 as the introduction of prazosin, followed by the development of various $\alpha_{1}$ adrenergic receptor antagonists, such as tamsulosin, terazosin, naftopidil ${ }^{13}$. Naftopidil 
is an $\alpha_{1}$-adrenergic receptor antagonist, which was marketed in Japan since 1999, acting by relaxation of vascular smooth muscle ${ }^{14}$. A widely accepted model of adrenergic ligands provides the presence of an arylpiperazine moiety, a linker and a heterocycle system (Figure 1). Many studies have highlighted the arylpiperazine moiety as the key pharmacophore of $\alpha_{1}$-adrenergic receptor antagonists, which consists a positive ionizable pharmacophore features. Besides, the isopropoxy moiety has also revealed a key feature of $\alpha_{1}$-adrenergic receptor antagonist as a hydrogen bond receptor ${ }^{15}$.

Based on the reported model of $\alpha_{1}$-adrenergic receptor antagonist and our previous studies about XJP ${ }^{16}$, we envisioned the hybrids of naftopidil and XJP would be potent antihypertensive hybrids with $\alpha_{1}$-adrenergic receptor antagonistic activity and cardioprotective activities. Thus, as shown in Figure 1, the hybrids 6a-r were designed by replacing the naphthalene moiety of naftopidil with naturally occurring heterocycle isochromanone-4-one (XJP). The group linked to the piperazine nucleus was explored by introducing substituted aryl and alkyl while the key positive ionizable center and hydrogen bond acceptor were reserved.

Herein, we reported the design, synthesis and pharmacological profile of a series of novel hybrids of XJP and naftopidil as antihypertensive candidates. Their vasodilation potency and $\alpha_{1}$-adrenergic receptor antagonistic activity in vitro were evaluated. Furthermore, the in vivo antihypertensive evaluation of the most potent compound $\mathbf{6 e}$ was performed in SHRs. 


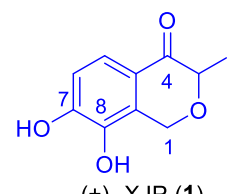

( \pm ) -XJP (1)

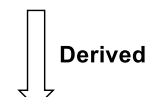

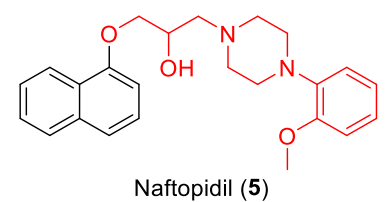

SARs

Positive lonizable
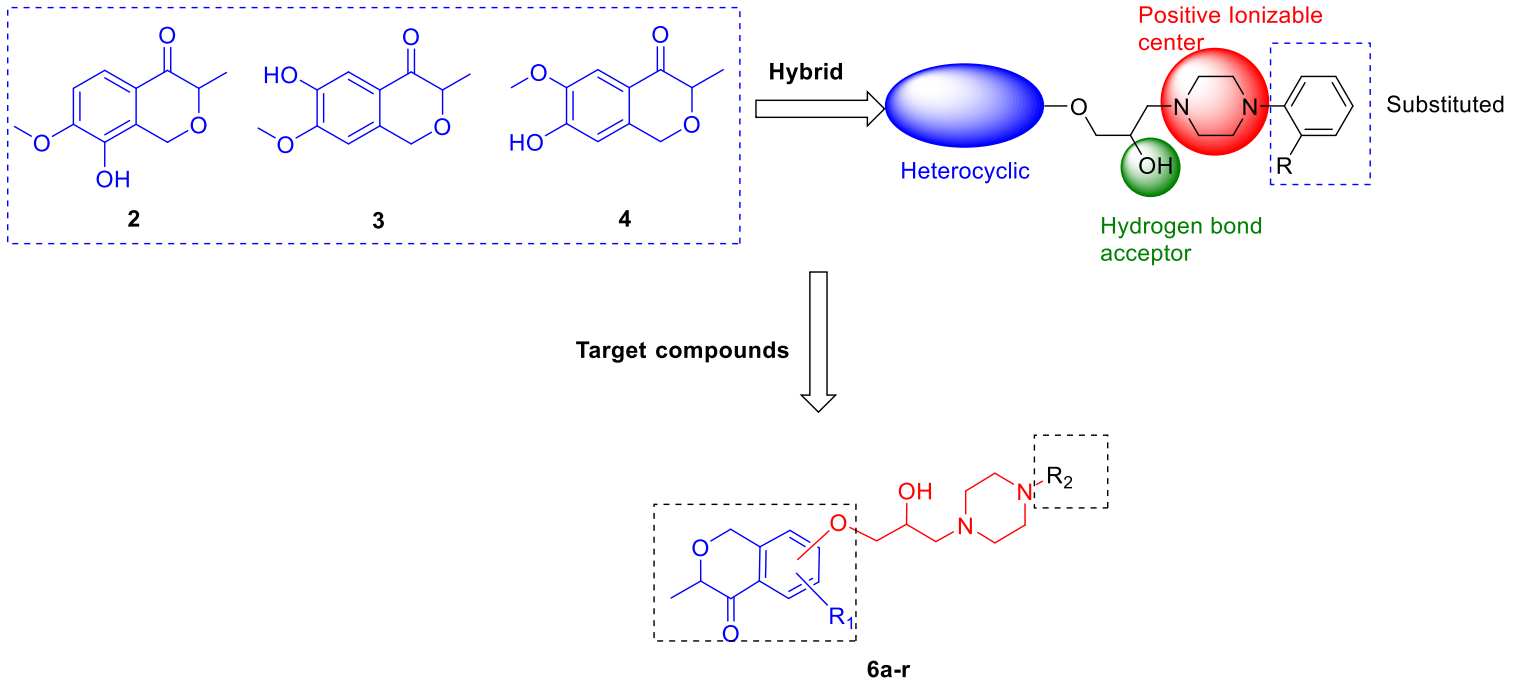

Figure 1. Strategy for the design of isochroman-4-one hybrids bearing piperazine moiety as antihypertensive agents.

\section{Result and discussion}

\subsection{Chemistry}

The traditional methods for the preparation of 4-isochroman-ones often suffered from limitations such as low yields, multi-step reactions or expensive metal catalysts ${ }^{15}$. An efficient synthesis of 4-isochroman-ones via Parham-type cyclization with Weinreb amide was developed by our group previously ${ }^{17}$. As shown in Scheme 1, the free phenolic hydroxyl groups of 7a-c were protected by benzyl group in quantitative yields, followed by reduction with sodium borohydride to provide alcohol 9a-c. Subsequent condensation with ethyl 2-bromopropionate in the presence of $\mathrm{NaH}$ afforded esters, which were hydrolyzed to get the acids 10a-c. The key intermediates 11a-c bearing Weinreb amide were prepared by alkylation with the $N, O$-dimethylhydroxylamine in $75-80 \%$ yields. The annulation proceeded smoothly by adding $t$-BuLi at $-78{ }^{\circ} \mathrm{C}$ within 1 min to afford the isochroman-ones 12a-c in $90-93 \%$ yields, which were then 
converted to the analogues of XJP (2-4) via a deprotection operation with $\mathrm{Pd} / \mathrm{C}$.

The synthetic route of the target compounds 6a-r was depicted in Scheme 2. Compounds 2-4 were treated with epichlorohydrin in the presence of potassium carbonate to give corresponding epoxides 13-15. Subsequent ring opening of the epoxides with various substituted piperazines afforded the target compounds 6a-r in $60-80 \%$ yields.
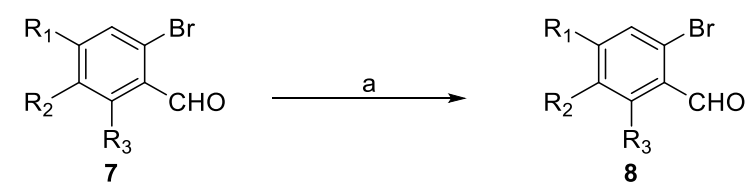

8a) $\mathrm{R}_{1}=\mathrm{OBn} \mathrm{R}_{2}=\mathrm{OCH}_{3} \mathrm{R}_{3}=\mathrm{H}$

7a) $\mathrm{R}_{1}=\mathrm{OH} \mathrm{R}=\mathrm{OCH}_{3} \mathrm{R}_{3}=\mathrm{H}$

7b) $\mathrm{R}_{1}=\mathrm{OCH}_{3} \mathrm{R}_{2}=\mathrm{OH} \mathrm{R}=\mathrm{H}$

7c) $\mathrm{R}_{1}=\mathrm{HR} \mathrm{R}_{2}=\mathrm{OCH}_{3} \mathrm{R}_{3}=\mathrm{OH}$

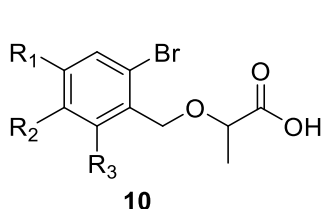

10a) $\mathrm{R}_{1}=\mathrm{OBn} \mathrm{R}_{2}=\mathrm{OCH}_{3} \mathrm{R}_{3}=\mathrm{H}$

10b) $\mathrm{R}_{1}=\mathrm{OCH}_{3} \mathrm{R}_{2}=O B n \mathrm{R}_{3}=\mathrm{H}$

10c) $\mathrm{R}_{1}=H \mathrm{R}_{2}=\mathrm{OCH}_{3} \mathrm{R}_{3}=\mathrm{OBn}$

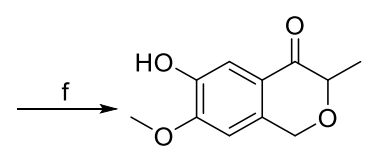

2<smiles>COc1cc2c(cc1O)COC(C)C2=O</smiles>

3 8b) $R_{1}=O C H_{3} R_{2}=O B n R_{3}=H$

8c) $R_{1}=H R_{2}=O C H_{3} R_{3}=O B n$

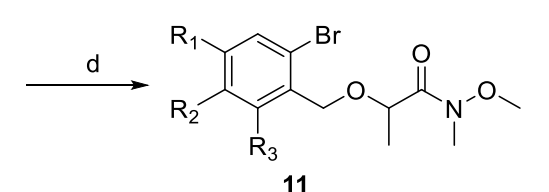

11a) $R_{1}=O B n R_{2}=O C H_{3} R_{3}=H$

11b) $R_{1}=O \mathrm{OCH}_{3} \mathrm{R}_{2}=O B n \mathrm{R}_{3}=\mathrm{H}$

11c) $R_{1}=H R_{2}=O C H_{3} R_{3}=O B n$

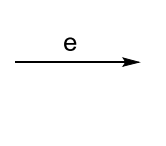

12a) $R_{1}=O B n R_{2}=O C H_{3} R_{3}=H$

12b) $\mathrm{R}_{1}=\mathrm{OCH}_{3} \mathrm{R}_{2}=\mathrm{OBn} \mathrm{R}_{3}=\mathrm{H}$

12c) $R_{1}=H R_{2}=O C H_{3} R_{3}=O B n$ 9a) $\mathrm{R}_{1}=\mathrm{OBn} \mathrm{R}_{2}=\mathrm{OCH}_{3} \mathrm{R}_{3}=\mathrm{H}$

9b) $\mathrm{R}_{1}=\mathrm{OCH}_{3} \mathrm{R}_{2}=\mathrm{OBn} \mathrm{R}_{3}=\mathrm{H}$

9c) $\mathrm{R}_{1}=H \mathrm{R}_{2}=\mathrm{OCH}_{3} \mathrm{R}_{3}=\mathrm{OBn}$<smiles>COc1ccc2c(c1O)COC(C)C2=O</smiles>

4

Scheme 1 Reagents and conditions: (a) $\mathrm{K}_{2} \mathrm{CO}_{3}$, benzyl bromide, DMF, $60^{\circ} \mathrm{C}$, overnight; (b) $\mathrm{NaBH}_{4}$, $\mathrm{MeOH}$, rt, 30 min, 93-96\% yields; (c) 1) Ethyl 2-bromopropionate, $\mathrm{NaH}$, rt, dry DMF; 2) $\mathrm{NaOH}$, $\mathrm{MeOH} / \mathrm{H}_{2} \mathrm{O}$, reflux, $30 \mathrm{~min}$; (d) Oxalyl chloride, then $N, O$-Dimethylhydroxylamine hydrochloride, 75-80\% yields; (e) $t$ - $\mathrm{BuLi},-78{ }^{\circ} \mathrm{C}, 1 \mathrm{~min}$, then $\mathrm{H}_{2} \mathrm{O}, 90-93 \%$ yields; (f) $\mathrm{Pd}-\mathrm{C} / \mathrm{H}_{2}$, THF, rt, 4 h, 64$72 \%$ yields. 


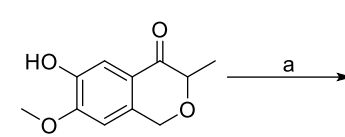

2
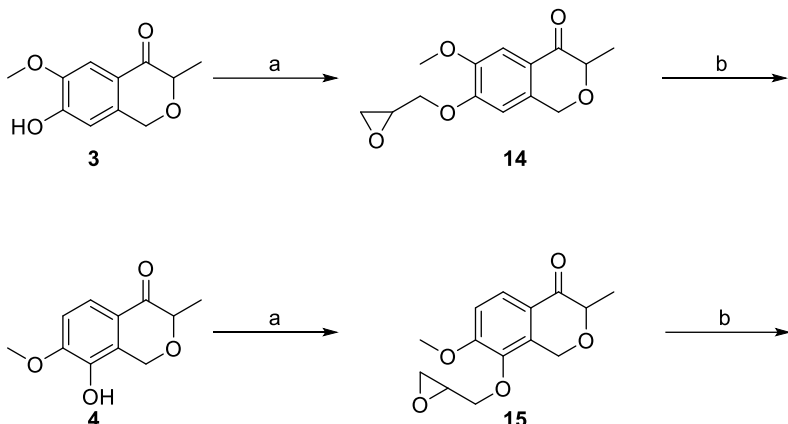<smiles>COc1cc2c(cc1OCC1CO1)C(=O)C(C)OC2</smiles>

13

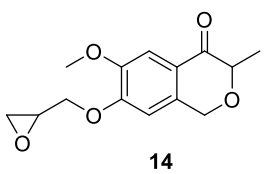

14
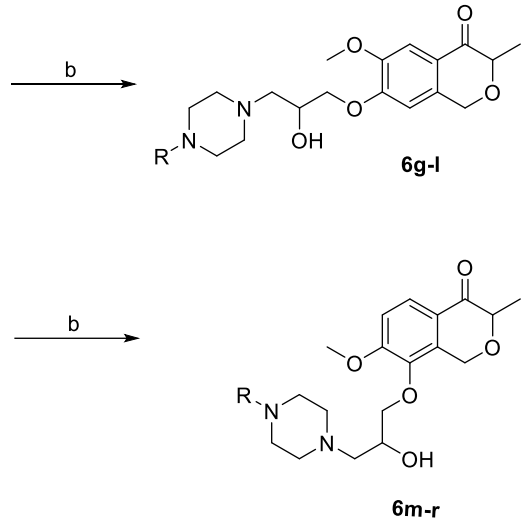

6a, 6g, 6m: R=phenyl

6b, 6h, 6n: R=2-methoxyphenyl

6c, 6i, 6o: R=2-ethoxyphenyl

6d, 6j, 6p: $R=2$-chlorophenyl

6e, 6k, 6q: R=tetrahydrofuran-2-carbonyl

6f, 6I, 6r: R=furan-2-carbonyl

Scheme 2 Reagents and conditions: (a) anhydrous acetone, epichlorohydrin, $\mathrm{K}_{2} \mathrm{CO}_{3}$, reflux, $75-82 \%$ yield; (b) $\mathrm{Zn}\left(\mathrm{ClO}_{4}\right)_{2}, \mathrm{DCM}, 80^{\circ} \mathrm{C}, 15 \mathrm{~min}, 60-80 \%$ yield.

\subsection{Pharmacological evaluation}

\subsubsection{In vitro antihypotensive activities}

Noradrenaline (NA)-induced isolated thoracic aorta ring was used to evaluate the in vitro antihypotensive effect of synthetic hybrids ${ }^{18}$. As shown in Table 1 , the vasodilation potency of target compounds 6a-r at concentrations of 10 and $100 \mu \mathrm{M}$ was preliminarilyobtained in an organ bath system. The most potent compound $\mathbf{6 e}$ exhibited the similar vasodilation activity compared with positive control naftopidil. The analysis of structure-activity relationship suggested that the substituted position of linker has influence on the vasodilation potency. Generally, compounds bearing substitutions at C8-position were more potent than corresponding analogues bearing substitutions at C6- and C7-positions. Interestingly, the tetrahydrofuran group was more favorable as the substitution linked to the piperazine moiety, while furan was unfavorable. Besides, the substituents on the phenyl group also affected the vasodilation potency, such as compounds 6m vs 6p. 
Table 1. The effects of target compounds on NA-induced contractions in isolated thoracic aorta rings.

\begin{tabular}{ccccccc}
\hline & \multicolumn{2}{c}{ Inhibition rate $(\%)$} & & \multicolumn{2}{c}{ Inhibition rate (\%) } \\
\cline { 2 - 3 } \cline { 6 - 7 } Compounds & $10 \mu \mathrm{M}$ & $100 \mu \mathrm{M}$ & Compounds & $10 \mu \mathrm{M}$ & $100 \mu \mathrm{M}$ \\
\hline $\mathbf{6 a}$ & $28.59 \pm 8.06$ & $46.35 \pm 18.56$ & $\mathbf{6 k}$ & $33.91 \pm 26.63$ & $40.12 \pm 27.35$ \\
$\mathbf{6 b}$ & $23.15 \pm 10.80$ & $44.63 \pm 10.40$ & & $\mathbf{6}$ & $17.83 \pm 7.42$ & $22.80 \pm 10.64$ \\
$\mathbf{6 c}$ & $25.35 \pm 7.55$ & $55.27 \pm 17.64$ & $\mathbf{6 m}$ & $37.83 \pm 11.34$ & $59.72 \pm 17.51$ \\
$\mathbf{6 d}$ & $30.16 \pm 13.87$ & $42.41 \pm 16.15$ & & $\mathbf{6 n}$ & $13.58 \pm 6.35$ & $24.35 \pm 7.04$ \\
$\mathbf{6 e}$ & $41.59 \pm 6.27$ & $64.04 \pm 11.60$ & $\mathbf{6 o}$ & $23.10 \pm 12.36$ & $36.08 \pm 14.84$ \\
$\mathbf{6 f}$ & $33.35 \pm 15.35$ & $44.86 \pm 14.32$ & & $\mathbf{6 p}$ & $18.21 \pm 5.39$ & $29.06 \pm 11.64$ \\
$\mathbf{6 g}$ & $39.20 \pm 11.59$ & $48.13 \pm 16.62$ & & $\mathbf{6 q}$ & $31.36 \pm 7.10$ & $45.11 \pm 12.79$ \\
$\mathbf{6 h}$ & $41.67 \pm 11.79$ & $50.35 \pm 7.50$ & & $\mathbf{6 r}$ & $20.81 \pm 13.33$ & $31.91 \pm 14.43$ \\
$\mathbf{6 i}$ & $11.62 \pm 7.26$ & $23.32 \pm 13.27$ & Naftopidil & $47.53 \pm 16.81$ & $65.15 \pm 11.05$ \\
$\mathbf{6 j}$ & $27.01 \pm 7.04$ & $32.43 \pm 12.31$ & DMSO & $16.88 \pm 7.63$ & $20.16 \pm 9.67$ \\
\hline
\end{tabular}

2.2.2. Evaluating $\mathrm{p} A_{2}$ values of representative compounds

Representative compounds with good vasodilation potency were selected to test their $\alpha_{1}$-adrenergic receptor antagonistic activity relative to phenylephrine-reduced contractions. Antagonist efficacies were expressed as $p \mathrm{~A}_{2}$ values calculated according to: $\mathrm{p} A_{2}=-\log [\mathrm{B}]+\log (r-1)$, where $[\mathrm{B}]$ is concentration of test compound and $r$ is the ratio of concentrations of agonist required to generate $50 \%$ maximal response in the presence and absence of test compound. As shown in Table 2, the vasodilation potency of these compounds correlated well with their $\alpha_{1}$-adrenergic receptor antagonistic activity. The $\alpha_{1}$-adrenergic receptor antagonistic activity of compound $6 \mathbf{e}\left(\mathrm{p} A_{2}=6.724\right)$ is comparable to that of naftopidil $\left(\mathrm{p} A_{2}=6.884\right)$. 
Table 2. Antagonist affinities of representative compounds, expressed as $\mathrm{p} A_{2}$ values on isolated ratthoracic aorta rings.

\begin{tabular}{ccc}
\hline Compounds & $\mathrm{p} A_{2} \pm \mathrm{SEM}$ & (Slope $\pm \mathrm{SEM})$ \\
\hline $\mathbf{6 c}$ & $6.314 \pm 0.13$ & $0.86 \pm 0.03$ \\
$\mathbf{6 e}$ & $6.724 \pm 0.18$ & $0.81 \pm 0.05$ \\
$\mathbf{6 f}$ & $5.811 \pm 0.17$ & $1.2 \pm 0.06$ \\
$\mathbf{6 g}$ & $6.207 \pm 0.17$ & $0.99 \pm 0.04$ \\
$\mathbf{6 h}$ & $5.658 \pm 0.16$ & $0.87 \pm 0.03$ \\
$\mathbf{6 m}$ & $6.528 \pm 0.19$ & $1.1 \pm 0.05$ \\
$\mathbf{6 q}$ & $6.469 \pm 0.11$ & $0.94 \pm 0.03$ \\
Naftopidil & $6.844 \pm 0.09$ & $0.88 \pm 0.02$ \\
\hline
\end{tabular}

2.2.3. In vivo antihypertensive activity of compound $\mathbf{6 e}$

Based on the results of vasodilation potency of target compounds, the in vivo antihypertensive evaluation of the most potent compound 6e was performed in SHRs. After oral administration of the positive control naftopidil (80 mg/kg), and compound 6e $(80 \mathrm{mg} / \mathrm{kg})$, the blood pressure and the heart rate of SHRs were detected from $0-8 \mathrm{~h}$. Compound 6e significantly reduced the systolic and diastolic blood pressure in SHRs throughout the observation period (Fig. $2 \mathrm{a}$ and $2 \mathrm{~b}$ ), and have no observable effects on the basal heart rate (Fig. 2d). The mean arterial pressure (MAP) of SHRs treat with $6 \mathbf{e}$ was reduced by almost $23.7 \%$ at $4 \mathrm{~h}$, which was superior to that of naftopidil (16.1\%) at 2h (Fig. 2c), suggesting that compound 6e may possess better cardioprotective effects than naftopidil.

Table 3. Effects of compound 6e on blood pressure in SHRs ${ }^{a}$

\begin{tabular}{cccccccc}
\hline \multirow{2}{*}{ Groups } & Parameter & \multicolumn{7}{c}{ Time } \\
\cline { 3 - 8 } & & $0 \mathrm{~h}$ & $1 \mathrm{~h}$ & $2 \mathrm{~h}$ & $4 \mathrm{~h}$ & $6 \mathrm{~h}$ & $8 \mathrm{~h}$ \\
\hline & $\mathrm{SAP}(\mathrm{mmHg})$ & $186.10 \pm 4.55^{b}$ & $185.35 \pm 10.30$ & $189.20 \pm 12.31$ & $178.66 \pm 17.18$ & $179.36 \pm 18.33$ & $179.42 \pm 13.70$ \\
Control & DAP (mmHg) & $149.50 \pm 5.4$ & $147.33 \pm 9.8$ & $149.90 \pm 6.0$ & $136.12 \pm 5.3$ & $137.54 \pm 9.2$ & $140.61 \pm 10.3$ \\
& & & & & & & \\
& MAP(mmH) & $161.70 \pm 5.12$ & $160.00 \pm 9.97$ & $163.00 \pm 8.10$ & $150.30 \pm 9.26$ & $151.48 \pm 12.24$ & $153.55 \pm 11.43$
\end{tabular}




\begin{tabular}{|c|c|c|c|c|c|c|c|}
\hline & HR (BPM) & $413.17 \pm 10.5$ & $415.25 \pm 14.7$ & $413.20 \pm 15.2$ & $410.80 \pm 17.4$ & $411.12 \pm 19.1$ & $412.77 \pm 11.3$ \\
\hline \multirow{4}{*}{ NAF } & $\mathrm{SAP}(\mathrm{mmHg})$ & $181.92 \pm 15.8$ & $161.36 \pm 13.4$ & $148.13 \pm 10.5^{* *}$ & $153.65 \pm 14.2^{* *}$ & $158.22 \pm 13.7 *$ & $170.50 \pm 16.4$ \\
\hline & DAP (mmHg) & $152.14 \pm 12.4$ & $140.19 \pm 10.3$ & $129.85 \pm 10.1 * *$ & $134.31 \pm 9.6^{* *}$ & $139.57 \pm 8.5$ & $143.06 \pm 10.2$ \\
\hline & MAP(mmHg) & $162.07 \pm 13.53$ & $147.25 \pm 11.33$ & $135.94 \pm 10.23 * *$ & $140.76 \pm 11.13^{* *}$ & $145.79 \pm 10.23$ & $152.21 \pm 12.27$ \\
\hline & HR (BPM) & $415.15 \pm 13.1$ & $412.14 \pm 17.2$ & $406.12 \pm 11.5$ & $409.22 \pm 16.4$ & $411.19 \pm 14.1$ & $417.25 \pm 18.6$ \\
\hline \multirow{4}{*}{$6 e$} & $\mathrm{SAP}(\mathrm{mmHg})$ & $187.64 \pm 22.3$ & $169.21 \pm 20.9^{*}$ & $150.80 \pm 16.3^{* *}$ & $140.29 \pm 11.4^{* *}$ & $157.46 \pm 15.6^{*}$ & $170.38 \pm 17.0$ \\
\hline & $\mathrm{DAP}(\mathrm{mmHg})$ & $156.23 \pm 10.1$ & $144.15 \pm 13.6$ & $131.86 \pm 12.0^{* *}$ & $120.54 \pm 10.1 * *$ & $132.51 \pm 11.5^{*}$ & $141.89 \pm 12.0$ \\
\hline & MAP(mmHg) & $166.70 \pm 14.17$ & $152.50 \pm 16.03$ & $138.17 \pm 13.43^{* *}$ & $127.12 \pm 10.53^{* *}$ & $140.83 \pm 12.87 *$ & $151.39 \pm 13.67$ \\
\hline & HR (BPM) & $415.33 \pm 16.6$ & $409.30 \pm 12.3$ & $408.14 \pm 14.9$ & $405.15 \pm 20.2$ & $411.01 \pm 18.0$ & $413.16 \pm 13.7$ \\
\hline
\end{tabular}

${ }^{a}$ SAP: systolic arterial pressure; DAP: diastolic arterial pressure; MAP: mean arterial pressure; HR: heart rate.

${ }^{b}$ Data are expressed as mean $\pm \operatorname{SEM}(\mathrm{n}=8), * P<0.1, * * p<0.05$ as compared with the respective control.

a)

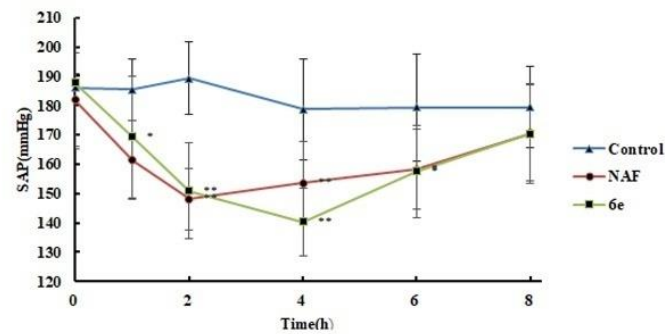

c)

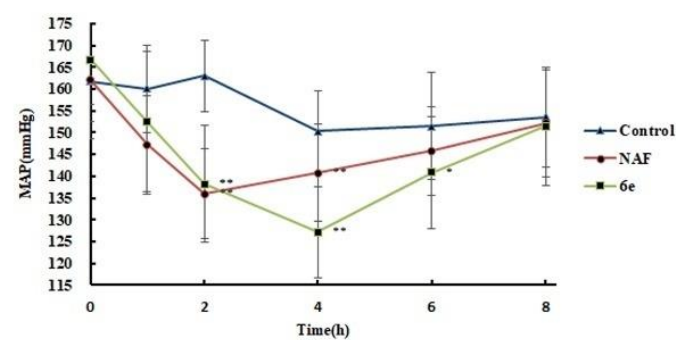

b)

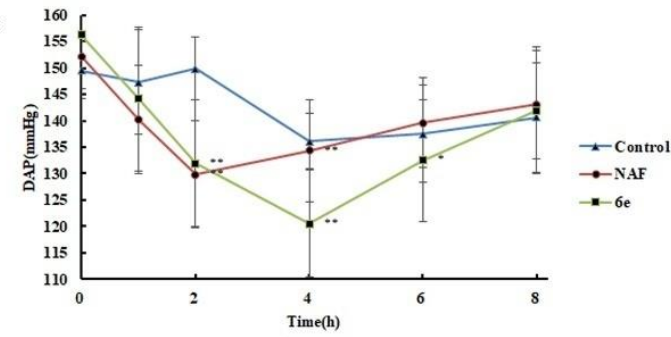

d)

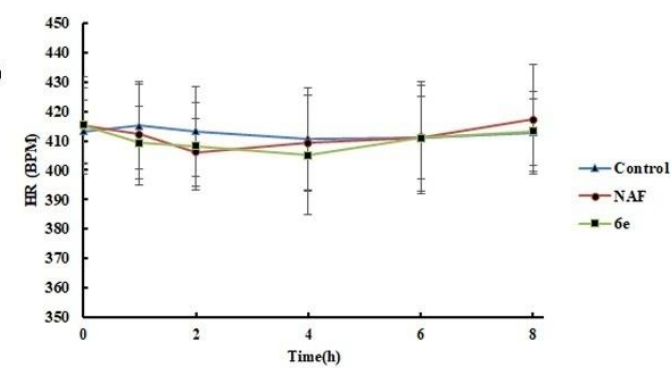

Figure 2. The acute antihypertensive activity of compound 6e in SHRs. (a) SAP: systolicarterial pressure ; (b) DAP: diastolic arterial pressure; (c) MAP:mean arterial pressure; (d) HR : heart rate. Data are presented as mean \pm SEM, $\mathrm{n}=8$. Significanceindicated by: $* \mathrm{P}<0.05$, $* * \mathrm{P}<0.01$, versus control.

\section{Conclusions}


In conclusion, based on our previous studies about the antihypertensive properties of XJP, a series of hybrids (6a-r) of XJP and $\alpha_{1}$-adrenergic receptor antagonist naftopidil were designed and synthesized to obtain new agents with stronger antihypertensive potency and safer profile. These novel hybrids bearing isochroman-4-one and arylpiperazine moieties exhibited moderate to good activities. Compounds $\mathbf{6 c}, \mathbf{6 e}, \mathbf{6 f}$, $\mathbf{6 g}, 6 \mathbf{h}, \mathbf{6 m}$ and $\mathbf{6 q}$, which have potent in vitro vasodilation potency, also exhibited good $\alpha_{1}$-adrenergic receptor antagonistic activity. Furthermore, the most potent compound $\mathbf{6 e}$ significantly reduced the systolic and diastolic blood pressure in SHRs and have no observable effects on the basal heart rate, which was comparable to that of naftopidil. The improved activities of these hybrid molecules qualified them as promising leads for the development of new antihypertensive agents.

\section{Experimental sections}

\subsection{Chemistry}

${ }^{1} \mathrm{H}$ NMR and ${ }^{13} \mathrm{C}$ NMR spectra were recorded on a BrukerAV-300NMR, deuterated solvents were $\mathrm{CDCl}_{3}$, DMSO- $d_{6}$ and MeOD- $d_{4}$. Mass spectra were obtained on an Agilent 1100-LC-MSD-Traps/SL. All reagents and solvents were commercially available and used without further purification. Anhydrous DMF was obtained by distillation over $\mathrm{CaH}_{2}$ and anhydrous THF was obtained by distillation over Na. Silica gel 60 H (200-300 mesh), manufactured by Qingdao Haiyang Chemical Group Co., Ltd (China) was used for general chromatography.

\subsubsection{General procedure for the synthesis of compounds 13,14 and 15 .}

Isochroman-4-one was dissolved in anhydrous acetone, $\mathrm{K}_{2} \mathrm{CO}_{3}$ was added to the solution. Epichlorohydrin was added after refluxing under nitrogen for $30 \mathrm{~min}$. The reaction was heated to reflux for $3 \mathrm{~h}$. After completion of the reaction, the filtrate was collected by suctioning and spinning. The filtrate was diluted with ethyl acetate and washed twice with water. The organic layers were washed with saturated brine and dried over anhydrous sodium sulfate, the crude product was purified by column chromatography to give white solid in $75-82 \%$ yields. 


\subsubsection{1.}

7-methoxy-3-methyl-6-(oxiran-2-ylmethoxy)isochroman-4-one (13)

White solid; ${ }^{1} \mathrm{H}$ NMR $\left(\mathrm{CDCl}_{3}, 300 \mathrm{MHz}\right) \delta: 7.51(\mathrm{~s}, 1 \mathrm{H}), 6.62(\mathrm{~s}, 1 \mathrm{H}), 4.85(\mathrm{~s}, 1 \mathrm{H})$, $4.33(\mathrm{~m}, 1 \mathrm{H}), 4.24(\mathrm{q}, J=6.7 \mathrm{~Hz}, 1 \mathrm{H}), 4.03(\mathrm{~m}, 1 \mathrm{H}), 3.93(\mathrm{~s}, 3 \mathrm{H}), 3.40(\mathrm{~m}, 1 \mathrm{H}), 2.92$ (m, 1H), $2.77(\mathrm{~m}, 1 \mathrm{H}), 1.50(\mathrm{~d}, J=6.7 \mathrm{~Hz}, 3 \mathrm{H})$; MS (ESI) $m / z: 265.1[\mathrm{M}+\mathrm{H}]^{+}$.

\subsubsection{2.}

\section{6-methoxy-3-methyl-7-(oxiran-2-ylmethoxy)isochroman-4-one(14)}

White solid; ${ }^{1} \mathrm{H}$ NMR $\left(\mathrm{CDCl}_{3}, 300 \mathrm{MHz}\right) \delta: 7.50$ (s, 1H), $6.68(\mathrm{~s}, 1 \mathrm{H}), 4.85(\mathrm{~s}, 2 \mathrm{H})$, $4.37(\mathrm{~m}, 1 \mathrm{H}), 4.22(\mathrm{q}, J=6.7 \mathrm{~Hz}, 1 \mathrm{H}), 4.05(\mathrm{~m}, 1 \mathrm{H}), 3.92(\mathrm{~s}, 3 \mathrm{H}), 3.41(\mathrm{~m}, 1 \mathrm{H}), 2.93$ $(\mathrm{m}, 1 \mathrm{H}), 2.78(\mathrm{~m}, 1 \mathrm{H}), 1.51(\mathrm{~d}, J=6.7 \mathrm{~Hz}, 3 \mathrm{H}) ; \mathrm{MS}(\mathrm{ESI}) \mathrm{m} / z: 265.1[\mathrm{M}+\mathrm{H}]^{+}$.

\subsubsection{3.}

\section{7-methoxy-3-methyl-8-(oxiran-2-ylmethoxy)isochroman-4-one (15)}

White solid; ${ }^{1} \mathrm{H}$ NMR $\left(\mathrm{CDCl}_{3}, 300 \mathrm{MHz}\right) \delta: 7.83(\mathrm{~d}, J=8.7 \mathrm{~Hz}, 1 \mathrm{H}), 6.93(\mathrm{~d}, J=8.7$ $\mathrm{Hz}, 1 \mathrm{H}), 5.20(\mathrm{q}, J=7.5 \mathrm{~Hz}, 1 \mathrm{H}), 4.82(\mathrm{~d}, J=15.7 \mathrm{~Hz}, 1 \mathrm{H}), 4.33(\mathrm{~m}, 1 \mathrm{H}), 4.19(\mathrm{q}, J=$ $6.7 \mathrm{~Hz}, 1 \mathrm{H}), 3.96(\mathrm{~s}, 3 \mathrm{H}), 3.93(\mathrm{~m}, 1 \mathrm{H}), 3.30(\mathrm{~m}, 1 \mathrm{H}), 2.88(\mathrm{~m}, 1 \mathrm{H}), 2.68(\mathrm{~m}, 1 \mathrm{H}), 1.49$ $(\mathrm{d}, J=6.7 \mathrm{~Hz}, 3 \mathrm{H}) ; \mathrm{MS}(\mathrm{ESI}) \mathrm{m} / \mathrm{z}: 265.1[\mathrm{M}+\mathrm{H}]^{+}$.

\subsubsection{General procedure for the synthesis of compounds 6a-r.}

The above-mentioned epoxy compound (13-15), substituted piperazine and $\mathrm{Zn}\left(\mathrm{ClO}_{4}\right)_{2}$ were mixed,dichloromethane was added, the reaction was heating to $80{ }^{\circ} \mathrm{C}$ in oil bath, The crude product was purified by column chromatography. The target compounds 6af, 6g-l, 6m-r were white solid in 60-80\% yields.

\subsubsection{1.}

\section{6-(2-hydroxy-3-(4-phenylpiperazin-1-yl)propoxy)-7-methoxy-3-} methylisochroman-4-on (6a)

${ }^{1} \mathrm{H}-\mathrm{NMR}\left(300 \mathrm{MHz}, \mathrm{CDCl}_{3}\right) \delta: 7.54$ (s, 1H), 7.28 (m, 2H), $6.91(\mathrm{~m}, 3 \mathrm{H}), 6.62(\mathrm{~s}, 1 \mathrm{H})$, $4.88(\mathrm{~s}, 2 \mathrm{H}), 4.26(\mathrm{~m}, 2 \mathrm{H}), 4.11(\mathrm{~m}, 2 \mathrm{H}), 3.94(\mathrm{~s}, 3 \mathrm{H}), 3.27(\mathrm{~s}, 4 \mathrm{H}), 2.91(\mathrm{~m}, 2 \mathrm{H}), 2.75-$ 
$2.63(\mathrm{~m}, 4 \mathrm{H}), 1.52(\mathrm{~d}, J=6.9 \mathrm{~Hz}, 3 \mathrm{H}) ;{ }^{13} \mathrm{C}-\mathrm{NMR}\left(75 \mathrm{MHz}, \mathrm{CDCl}_{3}\right) \delta: 194.76,154.56$, $151.17,148.02,137.42,129.12,122.40,119.84,116.12,110.13,106.21,169.72,154.36$, $147.79,137.37,122.19,109.90,106.18,77.96,71.85,66.53,65.71,60.38,56.12,53.37$, 49.21, 15.86; HRMS (ESI) calcd for $\mathrm{C}_{24} \mathrm{H}_{31} \mathrm{~N}_{2} \mathrm{O}_{5}[\mathrm{M}+\mathrm{H}]^{+}$427.2227, found 427.2231.

4.1.2.2.

6-(2-hydroxy-3-(4-(2-methoxyphenyl)piperazin-1-yl)propoxy)-7-methoxy-3methylisochroman-4-one (6b)

${ }^{1} \mathrm{H}-\mathrm{NMR}\left(300 \mathrm{MHz}, \mathrm{CDCl}_{3}\right) \delta: 7.39(\mathrm{~s}, 1 \mathrm{H}), 7.11(\mathrm{~d}, J=7.8 \mathrm{~Hz}, 1 \mathrm{H}), 7.01(\mathrm{~m}, 1 \mathrm{H})$, $6.84(\mathrm{~m}, 2 \mathrm{H}), 6.51(\mathrm{~s}, 1 \mathrm{H}), 4.77(\mathrm{~m}, 2 \mathrm{H}), 4.11(\mathrm{~m}, 3 \mathrm{H}), 3.94(\mathrm{~m}, 3 \mathrm{H}), 3.81(\mathrm{~s}, 3 \mathrm{H}), 3.78$ $(\mathrm{s}, 3 \mathrm{H}), 3.44-3.23(\mathrm{~m}, 7 \mathrm{H}), 3.05(\mathrm{~m}, 1 \mathrm{H}), 1.41(\mathrm{~d}, J=6.6 \mathrm{~Hz}, 3 \mathrm{H}) ;{ }^{13} \mathrm{C}-\mathrm{NMR}(75 \mathrm{MHz}$, $\left.\mathrm{CDCl}_{3}\right) \delta: 194.67,158.42,154.50,148.01,138.10,137.31,125.19,123.96,122.23$, 121.08, 111.83, 109.89, 106.17, 77.84, 71.47, 67.26, 66.47, 61.78, 57.36, 56.04, 55.38, 51.33, 44.67, 42.79, 15.79; HRMS (ESI) calcd for $\mathrm{C}_{25} \mathrm{H}_{31} \mathrm{~N}_{2} \mathrm{O}_{6} \mathrm{Na}_{2}[\mathrm{M}-\mathrm{H}+2 \mathrm{Na}]^{+}$ 501.1918, found 501.2232.

\subsubsection{3.}

6-(3-(4-(2-ethoxyphenyl)piperazin-1-yl)-2-hydroxypropoxy)-7-methoxy-3methylisochroman-4-one (6c)

${ }^{1} \mathrm{H}-\mathrm{NMR}\left(300 \mathrm{MHz}, \mathrm{CDCl}_{3}\right) \delta: 7.39(\mathrm{~s}, 1 \mathrm{H}), 7.11(\mathrm{~d}, J=7.5 \mathrm{~Hz}, 1 \mathrm{H}), 7.00(\mathrm{~m}, 1 \mathrm{H})$, $6.82(\mathrm{~m}, 2 \mathrm{H}), 6.51(\mathrm{~s}, 1 \mathrm{H}), 4.76(\mathrm{~m}, 2 \mathrm{H}), 4.14-3.94(\mathrm{~m}, 8 \mathrm{H}), 3.81(\mathrm{~s}, 3 \mathrm{H}), 3.42$ (q, $J=$ $8.1 \mathrm{~Hz}, 2 \mathrm{H}), 3.28(\mathrm{~m}, 5 \mathrm{H}), 3.05(\mathrm{~m}, 1 \mathrm{H}), 1.40(\mathrm{~m}, 6 \mathrm{H})$; HRMS (ESI) calcd for $\mathrm{C}_{26} \mathrm{H}_{33} \mathrm{~N}_{2} \mathrm{O}_{6} \mathrm{Na}_{2}[\mathrm{M}-\mathrm{H}+2 \mathrm{Na}]^{+}$515.2490, found 515.2401.

\subsubsection{4.}

\section{6-(3-(4-(2-chlorophenyl)piperazin-1-yl)-2-hydroxypropoxy)-7-methoxy-3-}

methylisochroman-4-one (6d)

${ }^{1} \mathrm{H}-\mathrm{NMR}\left(300 \mathrm{MHz}, \mathrm{CDCl}_{3}\right) \delta: 7.46(\mathrm{~s}, 1 \mathrm{H}), 7.38(\mathrm{~m}, 1 \mathrm{H}), 7.32(\mathrm{~m}, 1 \mathrm{H}), 7.23(\mathrm{~m}, 1 \mathrm{H})$, $7.03(\mathrm{~m}, 1 \mathrm{H}), 6.58(\mathrm{~s}, 1 \mathrm{H}), 4.85(\mathrm{~m}, 2 \mathrm{H}), 4.21(\mathrm{~m}, 1 \mathrm{H}), 4.13-4.01(\mathrm{~m}, 5 \mathrm{H}), 3.89(\mathrm{~s}, 3 \mathrm{H})$, 3.55-3.21 (m, 7H), $1.50(\mathrm{~d}, J=6.69,3 \mathrm{H})$; HRMS (ESI) calcd for $\mathrm{C}_{24} \mathrm{H}_{28} \mathrm{ClN}_{2} \mathrm{O}_{5} \mathrm{Na}_{2}$ $[\mathrm{M}-\mathrm{H}+2 \mathrm{Na}]^{+}$505.1838, found 505.1744.

\subsubsection{5.}

6-(2-hydroxy-3-(4-(tetrahydrofuran-2-carbonyl)piperazin-1-yl)propoxy)-7- 
methoxy-3-methylisochroman-4-one (6e)

${ }^{1} \mathrm{H}-\mathrm{NMR}\left(300 \mathrm{MHz}, \mathrm{CDCl}_{3}\right) \delta: 7.39(\mathrm{~s}, 1 \mathrm{H}), 6.52(\mathrm{~s}, 1 \mathrm{H}), 4.75(\mathrm{~s}, 2 \mathrm{H}), 4.51(\mathrm{t}, J=5.7$ $\mathrm{Hz}, 1 \mathrm{H}), 4.11$ (m, 2H), 3.98 (m, 2H), 3.9-3.7 (m, 5H), 3.60 (brs, 2H), 3.48 (brs, 2H), $2.5(\mathrm{~m}, 6 \mathrm{H}), 2.12(\mathrm{~m}, 1 \mathrm{H}), 2.0-1.7(\mathrm{~m}, 3 \mathrm{H}), 1.39(\mathrm{~d}, J=6.48 \mathrm{~Hz}, 3 \mathrm{H}) ;{ }^{13} \mathrm{C}-\mathrm{NMR}(75$ $\mathrm{MHz}_{\mathrm{CDCl}}$ ) $\delta: 194.60,169.72,154.36,147.79,137.37,122.19,109.90,106.18,75.63$, 71.74, 68.91, 66.36, 65.94, 60.36, 55.99, 53.64, 53.14, 53.00, 45.16, 41.78, 28.36, 25.56, 15.72; HRMS (ESI) calcd for $\mathrm{C}_{23} \mathrm{H}_{33} \mathrm{~N}_{2} \mathrm{O}_{7}[\mathrm{M}+\mathrm{H}]^{+} 449.2288$, found 449.2275.

\subsubsection{6.}

\section{6-(3-(4-(furan-2-carbonyl)piperazin-1-yl)-2-hydroxypropoxy)-7-methoxy-3-} methylisochroman-4-one (6f)

${ }^{1} \mathrm{H}-\mathrm{NMR}\left(300 \mathrm{MHz}, \mathrm{CDCl}_{3}\right) \delta: 7.45(\mathrm{~s}, 1 \mathrm{H}), 7.43(\mathrm{t}, J=0.78 \mathrm{~Hz}, 1 \mathrm{H}), 6.94(\mathrm{~d}, J=3.33$ $\mathrm{Hz}, 1 \mathrm{H}), 6.55(\mathrm{~s}, 1 \mathrm{H}), 6.42(\mathrm{t}, J=1.68 \mathrm{~Hz}, 1 \mathrm{H}), 5.25(\mathrm{~s}, 2 \mathrm{H}), 4.16(\mathrm{~m}, 2 \mathrm{H}), 4.12(\mathrm{~m}$, 2H), 3.85 (s, 3H), 3.77 (brs, 4H), 3.43 (brs. 1H), 2.67 (m, 2H), 2.58 (m, 4H), 1.44 (d, J $=6.69 \mathrm{~Hz}, 3 \mathrm{H}) ; \mathrm{HRMS}(\mathrm{ESI})$ calcd for $\mathrm{C}_{23} \mathrm{H}_{29} \mathrm{~N}_{2} \mathrm{O}_{7}[\mathrm{M}+\mathrm{H}]^{+} 445.1975$, found 445.1977 .

\subsubsection{7.}

\section{7-(2-hydroxy-3-(4-phenylpiperazin-1-yl)propoxy)-6-methoxy-3-}

\section{methylisochroman-4-one (6g)}

${ }^{1} \mathrm{H}-\mathrm{NMR}\left(300 \mathrm{MHz}, \mathrm{CDCl}_{3}\right) \delta: 7.48(\mathrm{~s}, 1 \mathrm{H}), 7.26(\mathrm{t}, J=8.7 \mathrm{~Hz}, 2 \mathrm{H}), 6.99$ (m, 3H), $6.67(\mathrm{~s}, 1 \mathrm{H}), 4.84(\mathrm{~m}, 2 \mathrm{H}), 4.21(\mathrm{~m}, 2 \mathrm{H}), 4.09(\mathrm{~m}, 2 \mathrm{H}), 3.89(\mathrm{~s}, 3 \mathrm{H}), 3.21(\mathrm{~m}, 4 \mathrm{H}), 2.81$ $(\mathrm{m}, 2 \mathrm{H}), 2.62(\mathrm{~m}, 4 \mathrm{H}), 1.50(\mathrm{~d}, J=6.66 \mathrm{~Hz}, 3 \mathrm{H}) ;{ }^{13} \mathrm{C}-\mathrm{NMR}\left(75 \mathrm{MHz}, \mathrm{CDCl}_{3}\right) \delta$ : 194.900, 153.344, 151.122, 149.086, 136.785, 129.150, 122.788, 119.926, 116.130, 108.398, 107.643, 77.989, 71.717, 71.662, 66.496, 65.616, 60.388, 56.055, 53.361, 49.245, 15.886; HRMS (ESI) calcd for $\mathrm{C}_{24} \mathrm{H}_{31} \mathrm{~N}_{2} \mathrm{O}_{5}[\mathrm{M}+\mathrm{H}]^{+}$427.2233, found 427.2228.

\subsubsection{8.}

\section{7-(2-hydroxy-3-(4-(2-methoxyphenyl)piperazin-1-yl)propoxy)-6-methoxy-3-}

\section{methylisochroman-4-one (6h)}

${ }^{1} \mathrm{H}-\mathrm{NMR}\left(300 \mathrm{MHz}, \mathrm{CDCl}_{3}\right) \delta: 7.22(\mathrm{~s}, 1 \mathrm{H}), 7.09(\mathrm{~d}, J=7.68 \mathrm{~Hz}, 1 \mathrm{H}), 7.00(\mathrm{t}, J=7.74$ $\mathrm{Hz}, 1 \mathrm{H}), 6.82(\mathrm{t}, J=7.38 \mathrm{~Hz}, 2 \mathrm{H}), 6.53(\mathrm{~s}, 1 \mathrm{H}), 4.73(\mathrm{~m}, 2 \mathrm{H}), 4.10(\mathrm{~m}, 3 \mathrm{H}), 3.96$ (m, $3 \mathrm{H}), 3.76(\mathrm{~d}, J=3.6 \mathrm{~Hz}, 6 \mathrm{H}), 3.40-3.05(\mathrm{~m}, 8 \mathrm{H}) ;{ }^{13} \mathrm{C}-\mathrm{NMR}\left(75 \mathrm{MHz}, \mathrm{CDCl}_{3}\right) \delta$ : 194.408, 158.054, 154.007, 152.907, 148.432, 136.385, 124.911, 123.630, 122.040, 
120.658, 114.407, 107.745, 107.049, 77.386, 70.934, 66.677, 65.914, 61.318, 56.610, 55.468, 54.921, 50.957, 44.090, 42.056, 15.330; HRMS (ESI) calcd for $\mathrm{C}_{25} \mathrm{H}_{31} \mathrm{~N}_{2} \mathrm{O}_{6} \mathrm{Na}_{2}$ $[\mathrm{M}-\mathrm{H}+2 \mathrm{Na}]^{+}$501.1978, found 501.2242.

\subsubsection{9.}

\section{7-(3-(4-(2-ethoxyphenyl)piperazin-1-yl)-2-hydroxypropoxy)-6-methoxy-3-} methylisochroman-4-one (6i)

${ }^{1} \mathrm{H}-\mathrm{NMR}\left(300 \mathrm{MHz}, \mathrm{CDCl}_{3}\right) \delta: 7.36(\mathrm{~s}, 1 \mathrm{H}), 7.10(\mathrm{~d}, J=7.71 \mathrm{~Hz}, 1 \mathrm{H}), 6.99(\mathrm{t}, J=6.5$ $\mathrm{Hz}, 1 \mathrm{H}), 6.80(\mathrm{t}, J=7.59 \mathrm{~Hz}, 2 \mathrm{H}), 6.54(\mathrm{~s}, 1 \mathrm{H}), 4.72(\mathrm{~m}, 2 \mathrm{H}), 4.13-3.94(\mathrm{~m}, 8 \mathrm{H}), 3.76$ (s, 3H), 3.10-3.04 (m, 7H), $3.07(\mathrm{~m}, 1 \mathrm{H}), 1.38(\mathrm{~m}, 6 \mathrm{H}) ;{ }^{13} \mathrm{C}-\mathrm{NMR}\left(75 \mathrm{MHz}, \mathrm{CDCl}_{3}\right) \delta$ : 194.4, 158.0, 153.56, 152.95, 148.49, 136.34, 125.08, 124.14, 122.05, 120.49, 112.10, $107.74,107.09,77.38,70.91,66.65,65.94,63.23,61.27,57.02,55.45,50.87,44.10$, 42.14, 15.33, 14.32; HRMS (ESI) calcd for $\mathrm{C}_{26} \mathrm{H}_{33} \mathrm{~N}_{2} \mathrm{O}_{6} \mathrm{Na}_{2}[\mathrm{M}-\mathrm{H}+2 \mathrm{Na}]^{+}$515.2134, found 515.2397.

\subsubsection{0.}

\section{7-(3-(4-(2-chlorophenyl)piperazin-1-yl)-2-hydroxypropoxy)-6-methoxy-3-} methylisochroman-4-one $(6 \mathbf{j})$

${ }^{1} \mathrm{H}-\mathrm{NMR}\left(300 \mathrm{MHz}, \mathrm{CDCl}_{3}\right) \delta: 7.46(\mathrm{~s}, 1 \mathrm{H}), 7.38(\mathrm{~m}, 1 \mathrm{H}), 7.32(\mathrm{~m}, 1 \mathrm{H}), 7.21(\mathrm{~m}, 1 \mathrm{H})$, $7.05(\mathrm{~m}, 1 \mathrm{H}), 6.60(\mathrm{~s}, 1 \mathrm{H}), 4.82(\mathrm{~s}, 2 \mathrm{H}), 4.21(\mathrm{~m}, 1 \mathrm{H}), 4.14-4.02(\mathrm{~m}, 5 \mathrm{H}), 3.87(\mathrm{~s}, 3 \mathrm{H})$, $1.50(\mathrm{~d}, J=6.69 \mathrm{~Hz}, 3 \mathrm{H}), 3.50-3.27(\mathrm{~m}, 8 \mathrm{H}) ;{ }^{13} \mathrm{C}-\mathrm{NMR}\left(75 \mathrm{MHz}, \mathrm{CDCl}_{3}\right) \delta: 194.93$, $158.49,153.22,148.91,146.71,136.88,130.77,127.73,125.57,125.06,122.65$, $108.27,107.63,77.92,71.30,67.37,66.42,61.74,55.99,54.95,51.45,44.30,42.23$, 15.84; HRMS (ESI) calcd for $\mathrm{C}_{24} \mathrm{H}_{28} \mathrm{ClN}_{2} \mathrm{O}_{5} \mathrm{Na}_{2} \quad[\mathrm{M}-\mathrm{H}+2 \mathrm{Na}]^{+}$505.1482, found 505.1747.

\subsubsection{1.}

7-(2-hydroxy-3-(4-(tetrahydrofuran-2-carbonyl)piperazin-1-yl)propoxy)-6methoxy-3-methylisochroman-4-one (6k)

${ }^{1} \mathrm{H}-\mathrm{NMR}\left(300 \mathrm{MHz}, \mathrm{CDCl}_{3}\right) \delta: 7.39(\mathrm{~s}, 1 \mathrm{H}), 6.58(\mathrm{~s}, 1 \mathrm{H}), 4.77(\mathrm{~m}, 2 \mathrm{H}), 4.50(\mathrm{t}, J=5.7$ $\mathrm{Hz}, 1 \mathrm{H}), 4.30(\mathrm{~m}, 2 \mathrm{H}), 4.02(\mathrm{~m}, 2 \mathrm{H}), 3.9-3.7(\mathrm{~m}, 5 \mathrm{H}), 3.65(\mathrm{~s}, 4 \mathrm{H}), 2.4-2.6(\mathrm{~m}, 6 \mathrm{H})$, $2.17(\mathrm{~m}, 1 \mathrm{H}), 1.90(\mathrm{~m}, 3 \mathrm{H}), 1.43(\mathrm{~d}, J=6.66 \mathrm{~Hz}, 3 \mathrm{H}) ;{ }^{13} \mathrm{C}-\mathrm{NMR}\left(75 \mathrm{MHz}, \mathrm{CDCl}_{3}\right) \delta$ : $194.31,169.29,152.69,148.46,136.29,122.25,107.86,107.13,77.40,75.27,71.09$, 
68.53, 65.89, 65.27, 59.89, 55.48, 44.68, 41.32, 27.85, 25.16, 15.30; HRMS (ESI) calcd for $\mathrm{C}_{23} \mathrm{H}_{33} \mathrm{~N}_{2} \mathrm{O}_{7}[\mathrm{M}+\mathrm{H}]^{+} 449.2288$, found 449.2285 .

4.1.2.12

7-(3-(4-(furan-2-carbonyl)piperazin-1-yl)-2-hydroxypropoxy)-6-methoxy-3methylisochroman-4-one (61)

${ }^{1} \mathrm{H}-\mathrm{NMR}\left(300 \mathrm{MHz}, \mathrm{CDCl}_{3}\right) \delta: 7.39(\mathrm{~d}, J=5.16 \mathrm{~Hz}, 2 \mathrm{H}), 6.92(\mathrm{~d}, J=3.27 \mathrm{~Hz}, 1 \mathrm{H})$, $6.58(\mathrm{~s}, 1 \mathrm{H}), 6.4(\mathrm{t}, J=1.56 \mathrm{~Hz}, 1 \mathrm{H}), 4.75(\mathrm{~s}, 2 \mathrm{H}), 4.15(\mathrm{~m}, 2 \mathrm{H}), 4.12(\mathrm{~m}, 2 \mathrm{H}), 3.84(\mathrm{~m}$, 7H), $2.55(\mathrm{~m}, 6 \mathrm{H}), 1.41(\mathrm{~d}, J=6.66 \mathrm{~Hz}, 3 \mathrm{H}) ;{ }^{13} \mathrm{C}-\mathrm{NMR}\left(75 \mathrm{MHz}, \mathrm{CDCl}_{3}\right) \delta: 194.32$, 158.50, 152.70, 148.48, 147.19, 143.28, 136.29, 122.27, 116.10, 110.82, 107.88, 107.16, $77.41,71.10,65.90,65.32,59.90,55.49,52.99$, 15.32; HRMS (ESI) calcd for $\mathrm{C}_{23} \mathrm{H}_{29} \mathrm{~N}_{2} \mathrm{O}_{7}[\mathrm{M}+\mathrm{H}]^{+}$445.1975, found 445.1979.

\subsubsection{3.}

\section{8-(2-hydroxy-3-(4-phenylpiperazin-1-yl)propoxy)-7-methoxy-3-}

\section{methylisochroman-4-one (6m)}

${ }^{1} \mathrm{H}-\mathrm{NMR}\left(300 \mathrm{MHz}, \mathrm{CDCl}_{3}\right) \delta: 7.85(\mathrm{~d}, J=8.7 \mathrm{~Hz}, 1 \mathrm{H}), 7.28(\mathrm{~m}, 2 \mathrm{H}), 6.94(\mathrm{~d}, J=$ $8.28 \mathrm{~Hz}, 3 \mathrm{H}), 6.88(\mathrm{t}, J=7.27 \mathrm{~Hz}, 1 \mathrm{H}), 5.25(\mathrm{~m}, 1 \mathrm{H}), 4.84(\mathrm{~m}, 1 \mathrm{H}), 4.20(\mathrm{q}, J=6.6$ Hz, 1H), 4.17-3.97 (m, 3H), 3.95 (s, 3H), 3.22 (m, 4H), 2.85 (m, 2H), 2.69-2.54 (m, $4 \mathrm{H}), 1.51(\mathrm{~d}, J=6.6 \mathrm{~Hz}, 3 \mathrm{H}) ;{ }^{13} \mathrm{C}-\mathrm{NMR}\left(75 \mathrm{MHz}, \mathrm{CDCl}_{3}\right) \delta: 194.93,156.54,151.15$, 136.08, 129.14, 124.16, 123.23, 119.91, 116.14, 111.08, 77.75, 75.43, 75.30, 66.29, $66.25,62.91,60.19,55.92,53.38,49.26,15.68$; HRMS (ESI) calcd for $\mathrm{C}_{24} \mathrm{H}_{31} \mathrm{~N}_{2} \mathrm{O}_{5}$ $[\mathrm{M}+\mathrm{H}]^{+}$427.2233, found 427.2223.

\subsubsection{4.}

8-(2-hydroxy-3-(4-(2-methoxyphenyl)piperazin-1-yl)propoxy)-7-methoxy-3methylisochroman-4-one (6n)

${ }^{1} \mathrm{H}-\mathrm{NMR}\left(300 \mathrm{MHz}, \mathrm{CDCl}_{3}\right) \delta: 7.72(\mathrm{~d}, J=8.4 \mathrm{~Hz}, 1 \mathrm{H}), 7.10(\mathrm{~d}, J=7.5 \mathrm{~Hz}, 1 \mathrm{H}), 7.03$ (m, 1H), $6.85(\mathrm{~m}, 3 \mathrm{H}), 5.08(\mathrm{~d}, J=15.6 \mathrm{~Hz}, 1 \mathrm{H}), 4.68(\mathrm{~m}, 1 \mathrm{H}), 4.06(\mathrm{~m}, 3 \mathrm{H}), 3.92(\mathrm{~m}$, 3H), $3.81(\mathrm{~s}, 3 \mathrm{H}), 3.78(\mathrm{~s}, 3 \mathrm{H}), 3.41-3.26(\mathrm{~m}, 7 \mathrm{H}), 3.02(\mathrm{~m}, 1 \mathrm{H}), 1.39$ (d, $J=6.6 \mathrm{~Hz}$, $3 \mathrm{H}) ;{ }^{13} \mathrm{C}-\mathrm{NMR}\left(75 \mathrm{MHz}, \mathrm{CDCl}_{3}\right) \delta: 194.43,158.02,156.02,154.09,141.74,135.49$, $125.01,123.75,123.44,122.155,120.65,111.39,110.56,77.09,74.69,74.57,67.29$, $62.33,61.33,56.97,55.35,54.94,50.90,44.09,42.02,15.11$; HRMS (ESI) calcd for 
$\mathrm{C}_{25} \mathrm{H}_{31} \mathrm{~N}_{2} \mathrm{O}_{6} \mathrm{Na} 2[\mathrm{M}-\mathrm{H}+2 \mathrm{Na}]^{+}$501.1978, found 501.2242.

4.1.2.15.

8-(3-(4-(2-ethoxyphenyl)piperazin-1-yl)-2-hydroxypropoxy)-7-methoxy-3methylisochroman-4-one (6o)

${ }^{1} \mathrm{H}-\mathrm{NMR}\left(300 \mathrm{MHz}, \mathrm{CDCl}_{3}\right) \delta: 7.73(\mathrm{~d}, J=8.7 \mathrm{~Hz}, 1 \mathrm{H}), 7.12(\mathrm{~d}, J=7.5 \mathrm{~Hz}, 1 \mathrm{H}), 7.02$ $(\mathrm{t}, J=7.56 \mathrm{~Hz}, 1 \mathrm{H}), 6.84(\mathrm{~m}, 3 \mathrm{H}), 5.10(\mathrm{~d}, J=15.6 \mathrm{~Hz}, 1 \mathrm{H}), 4.68(\mathrm{~m}, 1 \mathrm{H}), 4.11-3.85$ $(\mathrm{m}, 6 \mathrm{H}), 3.82(\mathrm{~s}, 3 \mathrm{H}), 3.44-3.27(\mathrm{~m}, 7 \mathrm{H}), 3.03(\mathrm{~m}, 1 \mathrm{H}), 1.41-1.36(\mathrm{~m}, 6 \mathrm{H}) ;{ }^{13} \mathrm{C}-\mathrm{NMR}$ $\left(75 \mathrm{MHz}, \mathrm{CDCl}_{3}\right) \delta: 194.46,156.06,153.68,141.77,135.52,125.06,124.19,123.47$, $122.57,120.50,112.08,110.54,77.12,74.67,74.54,67.33,67.28,63.24,62.34,61.27$, 57.30, 55.35, 50.81, 44.10, 42.14, 15.12, 14.34; HRMS (ESI) calcd for $\mathrm{C}_{26} \mathrm{H}_{33} \mathrm{~N}_{2} \mathrm{O}_{6} \mathrm{Na}_{2}$ $[\mathrm{M}-\mathrm{H}+2 \mathrm{Na}]^{+}$515.2134, found 515.2401.

4.1.2.16.

8-(3-(4-(2-chlorophenyl)piperazin-1-yl)-2-hydroxypropoxy)-7-methoxy-3methylisochroman-4-one (6p)

${ }^{1} \mathrm{H}-\mathrm{NMR}\left(300 \mathrm{MHz}, \mathrm{CDCl}_{3}\right) \delta: 7.82(\mathrm{~d}, J=8.67 \mathrm{~Hz}, 1 \mathrm{H}), 7.38(\mathrm{~m}, 2 \mathrm{H}), 7.25(\mathrm{~m}, 1 \mathrm{H})$, $7.06(\mathrm{~m}, 1 \mathrm{H}), 6.92(\mathrm{~d}, J=8.7 \mathrm{~Hz}, 1 \mathrm{H}), 5.15(\mathrm{~m}, 1 \mathrm{H}), 4.76(\mathrm{~m}, 1 \mathrm{H}), 4.19-4.03(\mathrm{~m}, 6 \mathrm{H})$, 3.99-3.88 (d, $J=5.97 \mathrm{~Hz}, 3 \mathrm{H}), 3.56-3.22(\mathrm{~m}, 8 \mathrm{H}), 1.48(\mathrm{~d}, J=6.69 \mathrm{~Hz}, 3 \mathrm{H}) ;{ }^{13} \mathrm{C}-$ NMR (75 MHz, $\left.\mathrm{CDCl}_{3}\right) \delta: 194.94,158.43,156.47,146.70,142.14,142.04,136.03$, $130.77,127.72,125.55,125.18,124.13,123.10,111.02,77.66,75.19,75.02,68.01$, 67.94, 62.80, 61.74, 55.90, 55.12, 51.11, 44.30, 42.22, 29.67, 15.64; HRMS (ESI) calcd for $\mathrm{C}_{24} \mathrm{H}_{28} \mathrm{ClN}_{2} \mathrm{O}_{5} \mathrm{Na}_{2}[\mathrm{M}-\mathrm{H}+2 \mathrm{Na}]^{+}$505.1482, found 505.1745.

4.1.2.17.

8-(2-hydroxy-3-(4-(tetrahydrofuran-2-carbonyl)piperazin-1-yl)propoxy)-7methoxy-3-methylisochroman-4-one (6q)

${ }^{1} \mathrm{H}-\mathrm{NMR}\left(300 \mathrm{MHz}, \mathrm{CDCl}_{3}\right) \delta: 7.74(\mathrm{~d}, J=8.64 \mathrm{~Hz}, 1 \mathrm{H}), 6.86(\mathrm{~d}, J=8.67 \mathrm{~Hz}, 1 \mathrm{H})$, $5.15(\mathrm{~m}, 1 \mathrm{H}), 4.76(\mathrm{~m}, 1 \mathrm{H}), 4.53(\mathrm{t}, J=5.88 \mathrm{~Hz}, 1 \mathrm{H}), 4.10(\mathrm{~m}, 1 \mathrm{H}), 4.01-3.73(\mathrm{~m}, 8 \mathrm{H})$, $3.63(\mathrm{~s}, 2 \mathrm{H}), 3.52(\mathrm{~s}, 2 \mathrm{H}), 2.50(\mathrm{~m}, 6 \mathrm{H}), 2.18(\mathrm{~m}, 1 \mathrm{H}), 1.93(\mathrm{~m}, 3 \mathrm{H}), 1.40(\mathrm{~d}, J=6.60$ $\mathrm{Hz}, 3 \mathrm{H}) ;{ }^{13} \mathrm{C}-\mathrm{NMR}\left(75 \mathrm{MHz}, \mathrm{CDCl}_{3}\right) \delta: 194.86,169.84,156.47,142.08,135.99,124.18$, $123.15,111.08,77.68,75.78,75.30,75.18,69.05,66.40,66.35,62.83,60.21,55.91$, 45.22, 41.85, 28.39, 25.67, 15.62; HRMS (ESI) calcd for $\mathrm{C}_{23} \mathrm{H}_{33} \mathrm{~N}_{2} \mathrm{O}_{7}[\mathrm{M}+\mathrm{H}]^{+} 449.2288$, 
found 449.2277 .

\subsubsection{8.}

\section{8-(3-(4-(furan-2-carbonyl)piperazin-1-yl)-2-hydroxypropoxy)-7-methoxy-3-}

\section{methylisochroman-4-one (6r)}

${ }^{1} \mathrm{H}-\mathrm{NMR}\left(300 \mathrm{MHz}, \mathrm{CDCl}_{3}\right) \delta: 7.73(\mathrm{~d}, J=8.37 \mathrm{~Hz}, 1 \mathrm{H}), 7.41(\mathrm{~s}, 1 \mathrm{H}), 6.92(\mathrm{~s}, 1 \mathrm{H})$, $6.85(\mathrm{~d}, J=8.37 \mathrm{~Hz}, 1 \mathrm{H}), 6.4(\mathrm{~s}, 1 \mathrm{H}), 5.15(\mathrm{~d}, J=4.68 \mathrm{~Hz}, 1 \mathrm{H}), 5.11(\mathrm{~d}, J=4.29 \mathrm{~Hz}$, 1H), 4.2-3.9 (m, 4H), 3.84 (s, 3H), 3.76 (brs, 4H), 2.49 (brs, 4H), 1.39 (d, J = 6.03 Hz, $3 \mathrm{H}) ;{ }^{13} \mathrm{C}-\mathrm{NMR}\left(75 \mathrm{MHz}, \mathrm{CDCl}_{3}\right) \delta: 194.85,159,01,156.47,147.69,143.78,142.08$, $141.97,135.99,128.74,124.14,123.12,116.56,111.30,111.08,77.66,75.34,75.22$, $66.50,66.44,62.82,60.21,55.90,53.49,15.61$; HRMS (ESI) calcd for $\mathrm{C}_{23} \mathrm{H}_{29} \mathrm{~N}_{2} \mathrm{O}_{7}$ $[\mathrm{M}+\mathrm{H}]^{+}$445.1975, found 445.1970.

\subsection{Pharmacological evaluation}

\subsubsection{Vasodilatory activity in thoracic aorta}

Male Sprague-Dawley rats (SD rats;180-200g body weight ) were narcotized by intraperitoneal injection of sodium pentobarbitone $(60 \mathrm{mg} / \mathrm{mL})$. The thoracic aorta was quickly excised and the connective and adipose tissues were cleaned from the vessel before it was cut into $4 \mathrm{~mm}$ segments, which were suspended in $10 \mathrm{~mL}$ organ baths. The baths were filled with gassed $\left(5 \% \mathrm{CO}_{2}\right.$ and $\left.95 \% \mathrm{O}_{2}\right)$ Tyrode's solution (in mmol/L: $\mathrm{NaCl} 112.2 ; \mathrm{KCl} 5.0 ; \mathrm{CaCl}_{2} 2.5 ; \mathrm{MgSO}_{4} 1.2 ; \mathrm{KH}_{2} \mathrm{PO}_{4} 1.0 ; \mathrm{NaHCO}_{3}$ 12.0; glucose 11.1) and warmed to $37^{\circ} \mathrm{C}$. The aortic rings were tensioned to $1.0 \mathrm{~g}$ and allowed toequilibrate for $1 \mathrm{~h}$.The isometric tension was recorded using a Powerlab 400TM data acquisition system (software Chart, version 7.0, AD Instruments, MA, USA). The preparation was allowed toequilibrate for at least $60 \mathrm{~min}$ prior to initiation of experimental procedures, and during this period, the incubation media was changed every $15 \mathrm{~min}$. After equilibration, the tissues were contracted twice by NA $(1 \mu \mathrm{M})$. When the contractions werestable, compound was added in progressively increasing cumulative concentrations $\left(10^{-8}-10^{-4} \mathrm{M}\right)$ at 30 min intervals. Data are expressed as mean \pm S.E.M. The compounds were dissolved in DMSO. DMSO had no effect on NE-contractile response. 


\subsubsection{The blocking activity $\left(\mathrm{p} A_{2}\right)$ of representative compounds}

The blocking activity $\left(\mathrm{p} A_{2}\right)$ of compound $6 \mathbf{e}, \mathbf{6 f}, \mathbf{6 g}, \mathbf{6 h}, \mathbf{6 m}$ and $\mathbf{6 q}$ was measured by using the methods similar to those described previously. Male rats (SPF rats; 200-250 g) were euthanasia by intraperitoneal injection of sodium pentobarbitone. The required organs were rapidly isolated and placed in Kreb's solution of the following composition (mM): $\mathrm{NaCl} 112.2 ; \mathrm{KCl} 5.0 ; \mathrm{CaCl}_{2} 2.5 ; \mathrm{MgSO}_{4}$ 1.2; $\mathrm{KH}_{2} \mathrm{PO}_{4}$ 1.0; $\mathrm{NaHCO}_{3}$ 12.0; glucose 11.1; respectively. After excess of fat and connective tissue was removed, the tissues were set up rapidly, under a suitable resting tension (vas thoracic aorta $1.0 \mathrm{~g}$ ) in 10 -mL organ baths containing Kreb's solution kept at $37{ }^{\circ} \mathrm{C}$ and aerated with $5 \% \mathrm{CO}_{2}$ : $95 \% \mathrm{O}_{2}$ at $\mathrm{PH}$ 7.4. Isolated vascular ring strips were denuded of endothelium to avoid any complicating effects of endothelium-derived factors. After equilibration for $1 \mathrm{~h}$ in the Krebs solution, concentration-response curves were constructed by cumulative addition of agonist NA. The concentration of NA in the organ bath was increased approximately three-fold at each step, with each addition being made only after the response to the previous addition had attained a maximal level and remained steady. The first concentrations-response curve was the basic one, and the other two with NA were repeated by adding testing compounds and naftopidil respectively. The individual tissues were exposed to only one tested antagonist. The $p \mathrm{~A} 2$ values of each compound were calculated from the following equation: $\mathrm{p} A_{2}=-\log (\mathrm{B})+\log (\mathrm{r}-1)$, where $(\mathrm{B})$ is the molar concentration of antagonists, and $\mathrm{r}$ is the ratio of agonist $\mathrm{EC}_{50}$ determined in the presence and absence of antagonist .

\subsubsection{Antihypertensive effects in SHRs}

Male SHRs were purchased from Vital River Laboratory Animal Technology Co. Ltd (Beijing, China). After one week of acclimation, 30 SHRs (200-250 g body weight) were randomly divided into 3 groups, namely the control group, captopril control group and the compounds 6egroups. After oral administration with saline water, captopril (80 $\mathrm{mg} / \mathrm{kg})$ and $6 \mathbf{e}(80 \mathrm{mg} / \mathrm{kg})$ to SHRs respectively, the SAP, DAP and heart rate (HR) were measured using the tail-cuff method with a blood pressure monitor (BP-2000, 
Visitech Systems, Inc., US) from 0 to $8 \mathrm{~h}$.

\section{Acknowledgements}

The authors acknowledge the National Natural Science Foundationof China (No. 81673306, 81703348, 81874289), the Project Program of State Key Laboratory of Natural Medicines, (No. SKLNMKF201710), and "Double First-Class" University project (No.CPU2018GY04 ), China Pharmaceutical University.

\section{References}

1. Hlatky MA, Greenland P, Arnett DK, Ballantyne CM, Criqui MH, Elkind MS, Go AS, Harrell FE Jr, Hong Y, Howard BV, Howard VJ, Hsue PY, Kramer CM, McConnell JP, Normand SL, O'Donnell CJ, Smith SC Jr, Wilson PW. Circulation. 2009; 119: 2408-16.

2. Mendis S, Davis S, Norrving B. Stroke. 2015; 46: E121-E122.

3. Guideline S. J hypertens. 1999; 17: 151-84.

4. Tang H, Walsh SP, Yan Y, de Jesus RK, Shahripour A, Teumelsan N, Zhu Y, Ha S, Owens KA, Thomas-Fowlkes BS, Felix JP, Liu J, Kohler M, Priest BT, Bailey T, Brochu R, Alonso-Galicia M, Kaczorowski GJ, Roy S, Yang L, Mills SG, Garcia ML, Pasternak A. ACS Med Chem Lett. 2012; 3: 367-72.

5. Menard PR, Suh JT, Jones H, Loev B, Neiss ES, Wilde J, Schwab A, Mann WS. J. Med. Chem. 1985; 28: 328-32.

6. Carosati E, Budriesi R, Ioan P, Ugenti MP, Frosini M, Fusi F, Corda G, Cosimelli B, Spinelli D, Chiarini A, Cruciani G. J. Med. Chem. 2008; 51: 5552-65.

7. Huang X, Xie L, Lin X, Su B. Anal Chem. 2017; 89: 945-951.

8. Reddy KS, Srinivasan N, Reddy CR, K N, Anjaneyulu Y, Venkatraman S, Bhattacharya A, Mathad VT. Org Process Res Dev. 2007; 11: 81-85.

9. Bai RR, Liu J, Zhu Y, Yang X, Yang C, Kong LY, Wang XB, Zhang HY, Yao HQ, Shen MQ, Wu XM, Xu JY. Bioorg Med Chem Lett. 2012;22: 6490-3.

10. Bai RR, Huang XJ, Yang X, Hong W, Tang YQ, Yao HQ, Jiang JY, Liu J, Shen MQ, Wu XM, Xu JY. Bioorg Med Chem. 2013; 21: 2495-502.

11. Liu J, Ren H, Xu JY, Bai RR, Yan Q, Huang WL, Wu XM, Fu JH, Wang QJ, Wu Q, Fu R. Biorg. Med. Chem. Lett. 2009; 19: 1822-24.

12. Bai RR, Xu ST, Liu J, Hong W, Tang YQ, Wu XM, Xie WJ, Yao HQ, Xu JY. Chin J Nat Med. 2013; 11: 538-45.

13. Lund-Johansen P, Hjermann I, Iversen BM, Thaulow E. Cardiology. 1993, 83: 1509.

14. Yamamoto M, Shimizu T, Shimizu S, Higashi Y, Nakamura K, Fujieda M, Saito M. J Pharmacol Sci. 2016, 132: 86-91.

15. Li MY, Xia L. Chem. Biol. Drug Des. 2007, 70: 461-64.

16. Bai RR, Yang X, Zhu Y, Zhou ZW, Xie WJ, Yao HQ, Jiang JY, Liu J, Shen MQ, 
Wu XM, Xu JY. Bioorg Med Chem. 2012, 20 (23), 6848-55.

17. Wang CL, Wu Z, Wang J, Liu J, Yao H, Lin AJ, Xu JY. Tetrahedron. 2015, 71: 8172-8177.

18. Connolly K, Jackson D, Pullen C, Fenning A. J Integr Med. 2015, 13 : 194-200. 